\title{
Influence du milieu d'extraction sur la détection du bois noir et de la flavescence dorée de la vigne, par des anticorps poly- et monoclonaux dirigés contre les phytoplasmes du stolbur et de la flavescence dorée
}

\author{
C Kuszala
}

Station de recherches sur les mycoplasmes et les arbovirus des plantes, Inra, BV 1540, F-21034 Dijon, France

(Reçu le 22 janvier 1996 ; accepté le 4 juin 1996)

\begin{abstract}
Résumé - En utilisant un anticorps monoclonal $(\mathrm{AcMc})$ dirigé contre le phytoplasme du stolbur dans un test Das-Elisa direct (double antibody sandwich enzyme-linked immunosorbent assay), nous avons détecté un antigène dans le jus brut de vigne présentant des symptômes de jaunisse pouvant être attribuée au bois noir (BN). La réactivité de l'AcMc est plus ou moins intense selon la nature du détergent utilisé pour l'extraction de l'antigène. Cet antigène est détecté chez au moins la moitié des vignes présentant des symptômes de jaunisse d'origine géographique diverse (France, Italie et Suisse). Cette réactivité fait de l'AcMc anti-stolbur un candidat pour le diagnostic de cette jaunisse. De plus, nous proposons un protocole d'extraction de vigne malade commun au test Elisa spécifique du phytoplasme du stolbur et au test Elisa spécifique du phytoplasme de la FD.
\end{abstract}

phytoplasme / bois noir / flavescence dorée / Elisa / détergent

Summary - Influence of extraction medium on Bois noir and Flavescence dorée détection, using polyclonal and monoclonal antibodies against stolbur and Flavescence dorée phytoplasmas. A double antibody sandwich enzyme-linked immunosorbent assay (DAS-ELISA) led us to show that a monoclonal antibody, specific for stolbur phytoplasma, recognized a 'Bois noir'-associated agent in extracts of diseased grapevines from different countries (France, Italy and Switzerland). In addition, we developed a common extraction procedure for the ELISA identification of both stolbur and Flavescence dorée phytoplasmas.

phytoplasma / Bois noir / Flavescence dorée / ELISA / detergent

\section{INTRODUCTION}

Les jaunisses de la vigne, attribuées à des phytoplasmes précédemment appelés MLO (mycoplasma-like organism), sont en progression constante dans les différentes régions viticoles du monde : en France (Caudwell et al, 1971b ; Daire et al, 1993c), en Allemagne (Gärtel, 1965), en Suisse (Cazelles et Kuszala, 1993), en Italie (Credi et Santucci, 1992), en Israël (Boubals, 1993), aux États-Unis (Pearson et al, 1985), en
Australie (Magarey et Wachtel, 1986). Le terme de jaunisse a été donné pour souligner le symptôme le plus frappant de ces maladies, bien que sur les cépages rouges on observe, au contraire un rougissement. Ce changement de coloration est considéré comme une manifestation secondaire provoquée par la perturbation des fonctions par le phloème. Elle est due d'une part à la disparition de la chlorophylle et des thylakoïdes qui la portent, et d'autre part à l'apparition de plastoglobules contenant des caroténoïdes dissous 
(Gourret, 1971). Ces maladies peuvent avoir une grande importance économique en raison de la diminution de la productivité et de la qualité du raisin. Certaines peuvent entraîner une baisse de vigueur et même la mort des pieds de vigne. Les agents responsables, les phytoplasmes, sont des microorganismes $(50-1000 \mathrm{~nm})$ sans paroi, appartenant à la classe des mollicutes. Ils sont localisés dans les tubes criblés du phloème des plantes qu'ils parasitent. À ce jour, et malgré de nombreuses tentatives, les phytoplasmes ne sont pas cultivés en milieu acellulaire (Sears et Schewe, 1994). Ces dernières années, l'approche de la biologie moléculaire par différents laboratoires a permis de progresser dans leur connaissance et de les distinguer des autres mollicutes. Les principaux travaux ayant motivé leur nouvelle appellation «phytoplasmes» ont été récemment relatés dans une revue (Cousin, 1995). II a été possible de les classer (Seemüller et al, 1994) et d'attribuer un nom à l'un d'entre eux (Zreik et al, 1995).

Actuellement, sous nos climats, les vignes sont contaminées principalement par deux phytoplasmoses : la flavescence dorée (FD) et le bois noir (BN).

La FD est bien connue (Caudwell et Ottenwaelter, 1957). Son étiologie a été démontrée par la microscopie électronique (Caudwell et al, 1971a) et par la transmission de vigne à vigne par le vecteur naturel Scaphoideus titanus Ball (Schwester et al, 1963). La sérologie et l'hybridation ADN-ADN utilisant des réactifs préparés à partir d'isolats entretenus au laboratoire dans des hôtes expérimentaux ont permis la détection directe dans la vigne : en sérologie (Caudwell et Kuszala, 1992) ou en biologie moléculaire (Daire et al, 1992).

Le type BN a été décrit en France dans la région de Bourgogne dès 1961 (Caudwell). En Allemagne, il a été signalé sous le terme de Vergilbungskrankheit (VK) par Gärtel en 1965 dans les vallées de la Moselle et du Rhin. II a également été mentionné en Suisse (Cazelles et al, 1992) et en Italie (Credi et Santucci, 1992). Le $\mathrm{BN}$ a été distingué de la FD du fait que la cicadelle $S$ titanus, vectrice de la FD, ne transmet pas le BN même expérimentalement (Caudwell et al, 1971b). Toutefois la similitude des symptômes avec la FD et la présence de particules de type mycoplasme (Granata et Grimaldi, 1991) ont permis de soutenir l'hypothèse que les agents pathogènes de ces jaunisses étaient également des phytoplasmes. La détection des phyto- plasmes en PCR (polymerase chain reaction) utilisant des amorces pour l'ADNr 165 suivie d'une analyse de restriction (RFLP : restriction fragment length polymorphism) a permis de montrer la présence d'un phytoplasme appartenant au même groupe ribosomique que le phytoplasme du stolbur dans des vignes atteintes de $\mathrm{BN}$ en France (Daire et al, 1993b), et dans divers pays : en Italie et en Israël (Daire et al, 1993c), en Suisse (Daire, communication personnelle), en Allemagne (Maixner et al, 1994).

L'utilisation de la PCR et de la RFLP des fragments amplifiés a permis de classer la FD, le BN et la VK par rapport à d'autres phytoplasmoses (Daire, 1994 ; Seemüller et al, 1994). En effet, les comparaisons de séquences d'ADNr $16 S$ indiquent que les isolats FD et les isolats de jaunisse de l'orme sont étroitement apparentés mais distincts, et que la VK est apparentée au stolbur. La séquence de l'espaceur intergénique 16S-23S du phytoplasme du BN montre qu'elle est identique à celle du stolbur (Daire, 1994). BN, stolbur et $V K$, forment un sous-groupe du groupe des jaunisses de l'aster (Kirkpatrick et al, 1994 ; Seemüller et al, 1994).

Parallèlement aux approches de la biologie moléculaire, la sérologie avec des anticorps polyclonaux ou monoclonaux (AcMc) dirigés contre les phytoplasmes de plusieurs jaunisses (Caudwell et al, 1982 ; Clark et al, 1988 ; Boudon-Padieu et al, 1989 ; Cousin et al, 1989 ; Schwartz et al, 1989 ; Garnier et al, 1990) a permis la détection et la caractérisation des phytoplasmes agents de maladies des plantes. Dans le cas de la FD, la mise au point du test Elisa (enzyme-linked immunosorbent assay) sur vignes malades a nécessité l'addition de détergents au milieu d'extraction pour assurer l'accessibilité aux antigènes du phytoplasme (Caudwell et Kuszala, 1992). Ainsi, les tests génomique (PCR) et sérologique (Elisa) sur la FD ont permis de préciser, en France et dans le monde, les zones de présence de la FD et des autres jaunisses comme le BN (Daire et al, 1993a ; Kuszala et al, 1993). En France, elles se superposent dans les vignobles du Sud.

La parenté entre le stolbur et l'agent du BN, au sein d'un même groupe ribosomique, a suggéré qu'une parenté sérologique pouvait également exister entre ces deux phytoplasmes. Le stolbur décrit par Valenta et al (1961) en Tchécoslovaquie, a également été décrit en France par Cousin et Grison (1966), puis étudié en fluorescence (Cousin et al, 1968). C'est une 
maladie à phytoplasme des solanacées, transmise par plusieurs cicadelles dont Hyalesthes obsoletus. Actuellement on constate une large distribution de cette maladie dans le monde. En France, un anticorps polyclonal contre le stolbur avait déjà été utilisé pour un diagnostic spécifique de la maladie en immunofluorescence (Cousin et al, 1989). Puis la production d'un AcMc dirigé contre le phytoplasme du stolbur (Garnier et al, 1990) a permis de le détecter par Elisa dans de nombreuses plantes hôtes : tomate, poivron, aubergine, tabac, lavandin et liseron, ce dernier jouant le rôle de plante réservoir. II a également été montré le rôle de $H$ obsoletus comme vecteur du stolbur en France (Fos et al, 1992). Récemment des $H$ obsoletus, capturés aux alentours des vignobles en Moselle (Allemagne), ont permis l'inoculation expérimentale d'un phytoplasme de type stolbur à des vignes saines (Maixner, 1994).

L'objet de cette étude a été, dans une première étape, de vérifier l'existence d'une parenté sérologique entre le phytoplasme du stolbur et l'agent associé au $\mathrm{BN}$, afin de permettre la détection du BN en Elisa, puis son étude épidémiologique. Dans une seconde étape, nous avons recherché un tampon d'extraction permettant la réalisation, sur le même extrait de vigne, du test Elisa spécifique du phytoplasme du stolbur et de celui spécifique du phytoplasme de la FD.

\section{MATÉRIEL ET MÉTHODES}

\section{Matériel végétal}

Les vignes (Vitis vinifera $L$ ) malades présentaient les symptômes typiques de jaunisse: sur feuillage : enroulement accompagné de jaunissement ou de rougissement du limbe, soit généralisé, soit sectoriel limité par les nervures ; sur bois : non-aoûtement des sarments de la base au sommet ; sur grappes : dessèchement de la rafle et des grains. Les échantillons des vignes malades poussées au vignoble étaient constitués de rameaux entiers provenant des régions contaminées de France : Alsace, Champagne, Bourgogne et Franche-Comté, de Suisse : Suisse romande et Tessin (fournis par O Cazelles) ; et d'Italie : Bologne (fournis par R Credi) et Sicile (fournis par G Granata), prélevés en septembre 1992 puis congelés. Ces échantillons ont répondu négativement au test Elisa spécifique FD (Kuszala et al, 1993). Nous avons comparé nos échantillons à des vignes témoins saines (fournies par l'Établissement national technique pour l'amélioration de la viticulture : Entav) et à des vignes témoins malades FD.
La souche de référence du phytoplasme de la FD sur pervenche de Madagascar (Catharanthus roseus $L$ ) et sur fève (Vicia faba L) provenait de notre collection et la souche de stolbur $\mathrm{C}$ de celle de MT Cousin (Inra, Versailles). Des pervenches et des fèves saines ont été utilisées comme témoins négatifs.

\section{Tampons d'extraction de l'agent associé au $B N$}

Au tampon de base habituellement utilisé pour la détection en Elisa du phytoplasme de la FD (Tris : $250 \mathrm{mM}$; $\mathrm{NaCl}: 137 \mathrm{mM}$; acide ascorbique : $30 \mathrm{mM}$; Polyclar AT : $4 \%$; pH : 8,2) (Caudwell et Kuszala, 1992), nous avons associé des détergents appartenant aux deux grands types : détergents ioniques (déoxycholate de sodium, sodium dodécyl sulfate, hexadecyl-trimethylammoniumbromid, Chaps), non ioniques (Hécameg, Triton $\times 100$, Tween 20, Tween 40, Tween 60, Tween 80, Brij 58, Tergitol Type NP 40, Nonidet P40). Dans le cas du Chaps (détergent convenant pour l'extraction de l'antigène du phytoplasme de la FD), nous avons parallèlement concentré des extraits par filtration moléculaire dans des concentrateurs Centrisart Sartorius : seuil de coupure 20000 Da. Les échantillons étaient constitués d'un même lot de feuilles de vigne (malade ou saine) découpés en fins fragments, mélangés et répartis aléatoirement pour chaque condition d'extraction (fig 1). Puis, nous avons fait une gamme de concentration avec les détergents les plus prometteurs (fig 2) avec le même tampon mais en remplaçant le Polyclar AT par du PVP K 30 à $5 \%$. Les échantillons ont été constitués de la même façon que dans la figure 1.

Pour optimiser le tampon d'extraction du BN, nous avons étudié l'effet de quelques agents tensio-actifs : polyvinylpyrrolidones solubles (PVP de différents poids moléculaires) en comparaison au Polyclar AT, à la concentration de $4 \%$. Nous avons utilisé le tampon de base, en remplaçant le Polyclar AT par l'agent tensioactif à étudier, en présence de Tween 40 à $3 \%$ (fig 3 ). Puis nous avons fait une gamme de concentration avec les polymères les plus prometteurs. Les échantillons ont été constitués de la même façon que dans la figure 1 .

\section{Tests comparatifs Elisa spécifique FD et Elisa spécifique stolbur selon l'hôte végétal}

Nous avons étudié l'effet de la présence d'un détergent (Tween 40, Chaps et Triton $X 100$ ) selon l'hôte végétal (vigne, pervenche ou fève) dans le tampon : Tris : $250 \mathrm{mM} ; \mathrm{NaCl}: 137 \mathrm{mM}$; PVP K $10: 7 \%$; pH : 8,2 (fig 4). 
DO $405 \mathrm{~nm}$

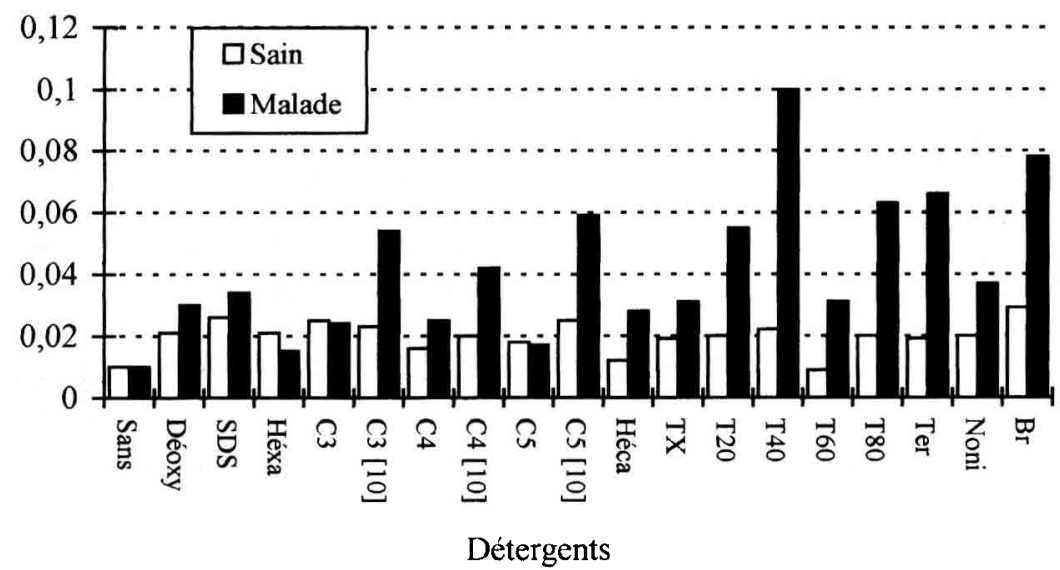

Fig 1. DO à $405 \mathrm{~nm}$ du test Elisa réalisé avec I'AcMc anti-stolbur sur extraits de feuilles de vigne saines ou infectées par le phytoplasme du BN obtenus en présence de divers détergents. Détergents ioniques : i) anioniques: Déoxy : Déoxycholate de $\mathrm{Na}: 0,3 \%$; SDS: sodium dodécyl sulfate : $0,3 \%$; ii) cationique : Hexa : Hexadecyltriméthyl-ammoniumbromide: $0,3 \%$; iii) zwitterionique : C3 : Chaps $3 \%$; C4 : Chaps $4 \%$; C5 : Chaps $5 \%$; [10] : extrait concentré 10 fois par ultrafiltration moléculaire. Détergents non ioniques Héca : Hécameg : $3 \%$; TX: Triton X100: $3 \%$; T20: Tween $20: 3 \%$; T40 : Tween $40: 3 \%$; T60 : Tween $60: 1 \%$; T80 Tween $80: 3 \%$; Ter: Tergitol Type NP $40: 3 \%$; Noni : Nonidet P $40: 3 \%$; $\mathrm{Br}$ : Brij $58: 3 \%$.

Fig 2. DO à $405 \mathrm{~nm}$ du test Elisa réalisé avec l'anticorps anti-stolbur et des échantillons de vignes saines ou infectées broyés en présence de différentes concentrations de détergent.

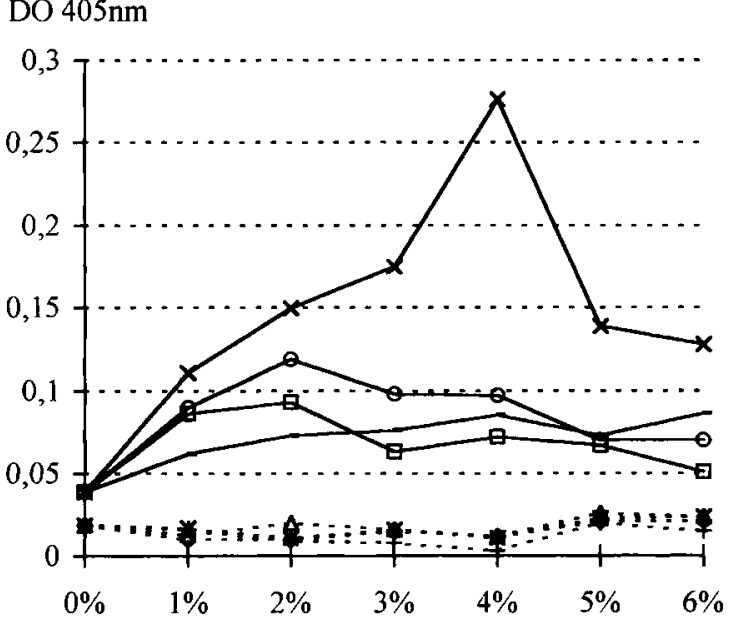

Détergents

DO $405 \mathrm{~nm}$

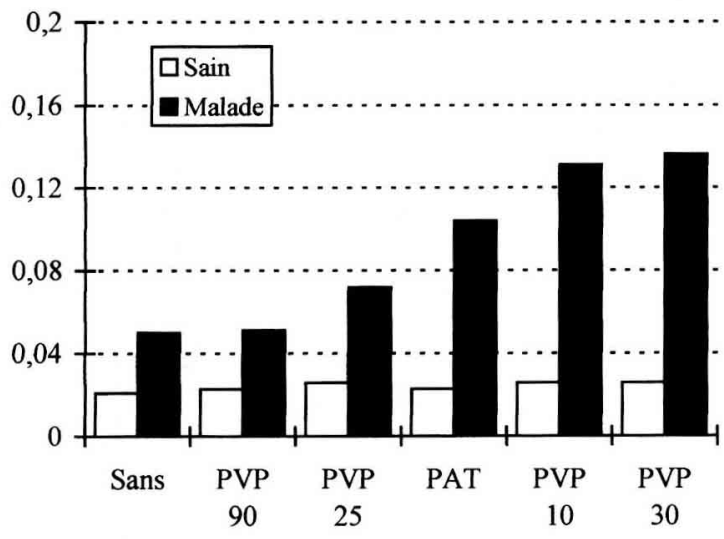

Polymères 4\%

Fig 3. DO à $405 \mathrm{~nm}$ du test Elisa réalisé avec l'anticorps antistolbur et des échantillons de vigne saines ou infectées broyés en présence de différents agents tensio-actifs. Agents tensioactifs : PAT : Polyclar AT ; PVP : polyvinylpyrrolidone (10 : poids moléculaire $10000 ; 25$ : poids moléculaire $24000 ; 30$ : poids moléculaire $40000 ; 90$ : poids moléculaire 360000 ).

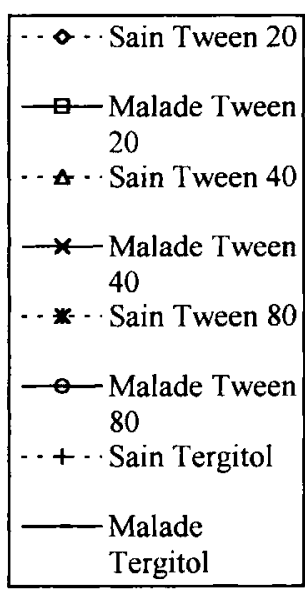

\section{Tests comparatifs Elisa spécifique FD et Elisa spécifique stolbur sur les mêmes extraits de plante}

Nous avons, en plus de ces tests comparatifs, voulu savoir si ce protocole permettait de détecter une double infection FD et BN (fig 5). Pour cela, nous avons broyé un mélange de feuilles de vigne contaminé par la FD et par le BN, $(0,250 \mathrm{~g}$ de chacun dans $5 \mathrm{~mL}$ de tampon). Dans cette étude, le tampon d'extraction pour les pervenches a été du Tris- $\mathrm{NaCl}$ (Tris : $250 \mathrm{mM} ; \mathrm{NaCl}: 137 \mathrm{mM}$ ), $\mathrm{pH}: 8,2$. Pour l'extraction des vignes, nous avons rajouté les produits suivants : PVP 10:7\%; acide ascorbique : $30 \mathrm{mM}$; Tween $40: 3 \% ; \mathrm{pH}: 8,2$.

\section{Préparation des échantillons}

Nous avons utilisé le broyeur à billes développé par Tecam pour Bioreba (Gugerli, 1984). Ce choix s'appuie sur nos résultats relatifs à la mise au point $\mathrm{du}$ 
Fig 4. Tests comparatifs Elisa spécifiques du phytoplasme du stolbur (a et b) et spécifiques du phytoplasme de la FD (c et d) sur extraits de vigne, de fève et de pervenche, en présence de différents détergents. a et c : lecture après trente minutes d'incubation du substrat.

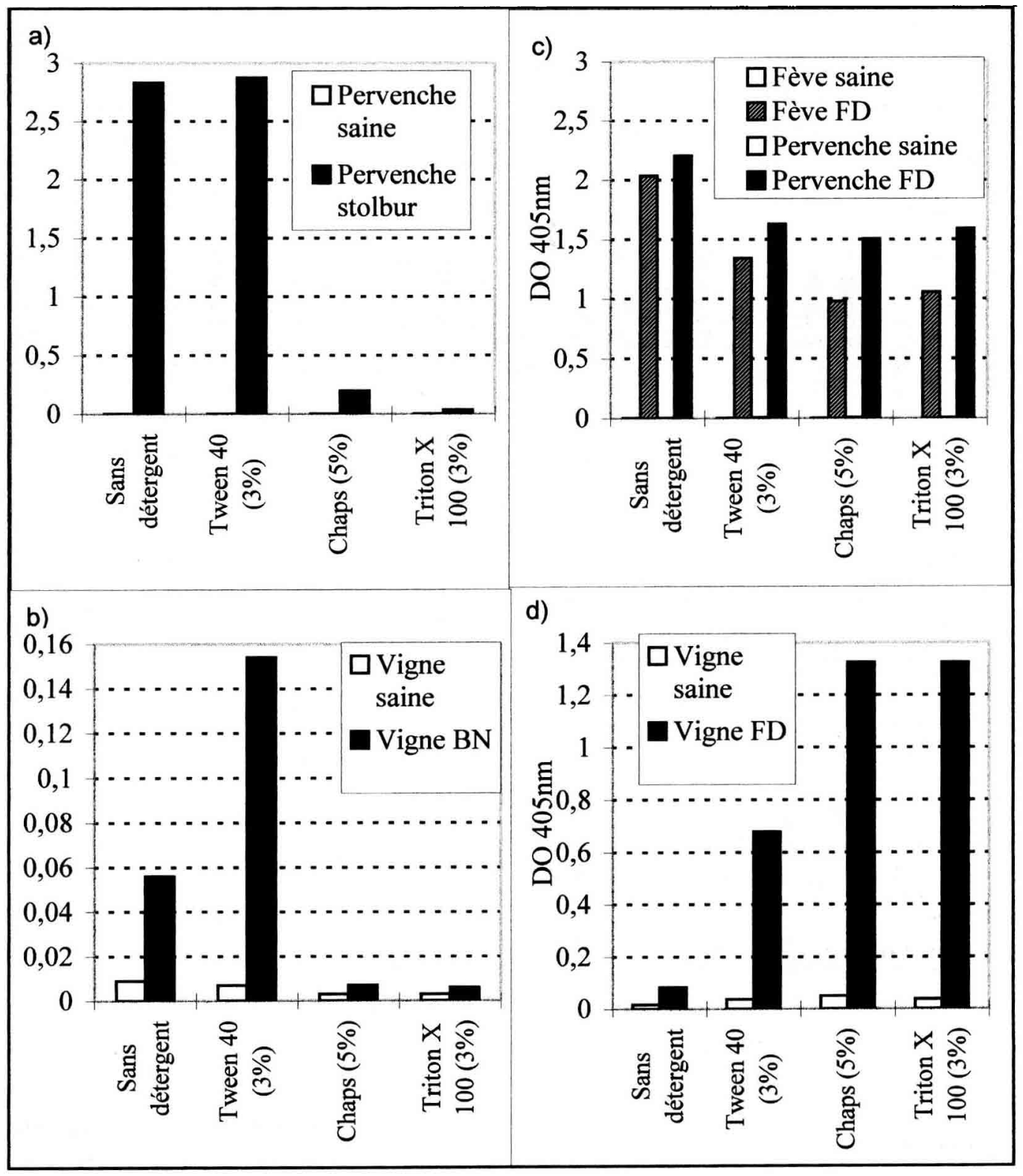

test Elisa-FD (Caudwell et Kuszala, 1992). Après broyage de $0,5 \mathrm{~g}$ de feuilles dans $5 \mathrm{~mL}$ de tampon d'extraction, l'extrait est centrifugé 5 minutes à $2900 \mathrm{~g}$ et le surnageant conservé.

\section{Techniques Das-Elisa (double antibody sand-} wich, enzyme-linked immunosorbent assay)

\section{Das-Elisa-direct spécifique du phytoplasme du stolbur}

Le test comporte quatre étapes :

- l'AcMc anti-stolbur (commercialisé par Sanofi, c'est celui produit par Garnier et al, 1990) dilué au 1/100e dans du tampon $\mathrm{Na}_{2} \mathrm{CO}_{3} \mathrm{pH} 9,6$, est adsorbé 2 heures à la température du laboratoire, sur une plaque de microtitration (Nunc Immuno Plate Maxisorp) ; ensuite les puits sont lavés avec du tampon Tris-caséine

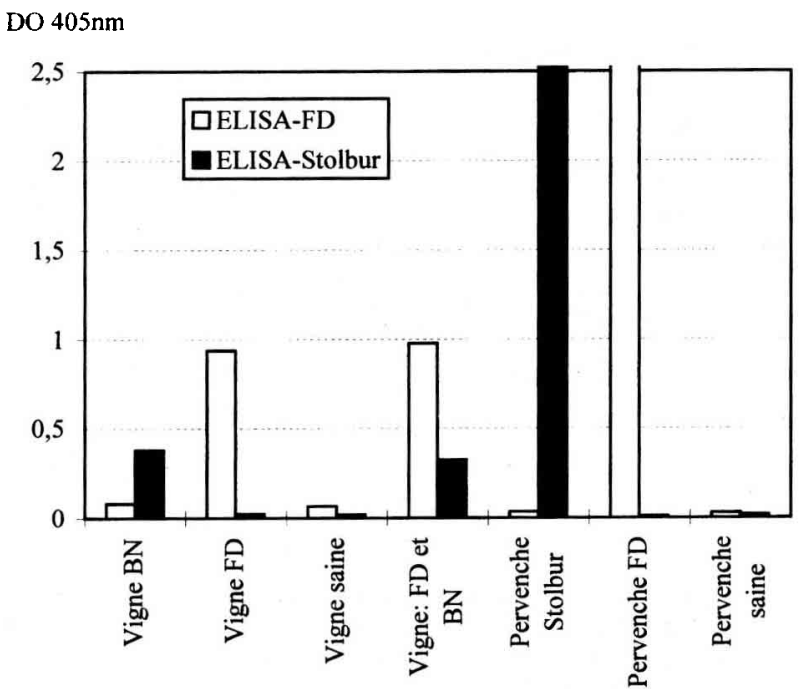

Fig 5. Application du test Elisa spécifique du phytoplasme de la FD et du test spécifique du phytoplasme du stolbur, aux mêmes extraits de plantes. 
(Tris : $10 \mathrm{mM} ; \mathrm{NaCl}: 150 \mathrm{mM}$; Caséine : 0,5\%) pH 7,6 et saturés avec le même tampon pendant 2 heures.

- Les échantillons sont déposés et mis en incubation pendant une nuit ; les puits sont ensuite lavés avec du tampon Tris-caséine $\mathrm{pH}$ 7,6.

- Le même AcMc conjugué à la phosphatase alcaline (Sanofi) dilué au $1 / 100^{\circ}$ dans du tampon Tris-caséine pH 7,6 est déposé sur la plaque. Après 2 heures d'incubation à la température du laboratoire les puits sont lavés avec du tampon Tris-caséine pH 7,6.

- La révélation est réalisée avec le paranitrophénylphosphate à $1 \mathrm{mg} / \mathrm{mL}$ dans du tampon diéthanolamine à $10 \%$ en présence de $\mathrm{MgCl}_{2}$ à $0,5 \mathrm{mM}: \mathrm{pH} 9,8$. L'absorbance est mesurée à $405 \mathrm{~nm}$ après deux heures trente d'incubation du substrat à température ambiante. Dans les cas particuliers nécessitant un temps d'incubation du substrat inférieur à deux heures trente, le temps utilisé est indiqué.

Les valeurs correspondent à la moyenne de deux puits par échantillon. On admet comme positifs les résultats lorsque la densité optique (DO) de l'échantillon de vigne malade est d'une part supérieure à deux fois celle de l'échantillon de vigne saine, et d'autre part supérieure à 0,1 .

\section{Das-Elisa-indirect spécifique du phytoplasme de la FD}

Le protocole utilisé est celui décrit par Caudwell et Kuszala (1992) sur vigne. II utilise en première couche des anticorps polyclonaux de lapin anti-fève FD (origine Inra-Dijon, Boudon-Padieu et al, 1989), 3,5 $\mu \mathrm{g} / \mathrm{mL}$ de tampon carbonate et comme anticorps révélateurs primaires un mélange de quatre surnageants de culture d'hybridomes anti-FD (origine Inra-Dijon, Schwartz et al, 1989) : 12, 17, 18 et 51 , suivis de l'anticorps secondaire de chèvre anti-souris conjugué à la phosphatase alcaline (Biosys BI2513CE/73).

\section{RÉSULTATS}

\section{Extraction de l'antigène de l'agent associé au $B N$ de la vigne}

\section{Effet des différents détergents}

Les résultats concernant l'influence de la nature et de la concentration du détergent pour l'extraction de l'antigène associé au BN, sont donnés dans la figure 1. En l'absence du détergent dans le milieu d'extraction, l'essai Elisa spécifique du phytoplasme du stolbur ne permet pas de différencier les plantes malades des plantes saines. Parmi les nombreux détergents utilisés, le Tween 40 est le plus efficace. D'autres détergents comme le Brij 58, le Tween 80, le Tween 20, le
Tergitol et le Chaps (ce dernier après concentration par ultrafiltration moléculaire des extraits) permettent aussi de distinguer les plantes saines des plantes infectées, mais la différence d'absorbance est insuffisante. Le tampon d'extraction utilisé pour le diagnostic de la FD n'est pas utilisable dans le cas du BN.

\section{Influence de la concentration en détergents}

La figure 2 montre les résultats de l'influence de la concentration en détergents dans le milieu d'extraction sur la détection de l'antigène. Les meilleurs résultats sont toujours obtenus avec le Tween 40 . Dès la concentration $1 \%$, l'échantillon malade se distingue nettement de l'échantillon sain. Nous constatons un pic d'absorbance à la concentration de $4 \%$. Dans des essais ultérieurs (non représentés), nous avons constaté que le pic pouvait se déplacer entre 2 et $4 \%$. Nous avons donc choisi la concentration $3 \%$ de Tween 40 .

\section{Choix d'un agent tensio-actif}

Les résultats sur le choix d'un agent tensio-actif (Polyclar AT ; PVP 10 ; PVP K 25 ; PVP K 30 et PVP K 90) en présence de Tween 40 sont donnés dans la figure 3 . À la dose de $4 \%$ le PVP 10 et le PVP K 30 donnent les meilleurs résultats. La présence d'un de ces agents tensio-actifs amplifie donc les résultats. Nous avons défini avec des gammes de concentrations de 0 à $7 \%$ (données non représentées) les meilleures concentrations à utiliser : $7 \%$ dans le cas du PVP 10 et $5 \%$ dans celui du PVP K 30 . Le Polyclar AT, sélectionné pour le tampon d'extraction de la FD, ne permet pas d'optimiser le tampon d'extraction du BN.

\section{Influence de l'hôte végétal sur la réponse Elisa en présence de détergents}

La comparaison de la réactivité de l'AcMc antistolbur, selon l'hôte végétal (pervenche ou vigne), est donnée dans la figure $4 a$ et $b$ (Elisa spécifique du phytoplasme du stolbur). En absence de détergent, seul l'extrait fait à partir de pervenche infectée réagit nettement avec l'AcMc anti-stolbur. En revanche, en présence de Tween 40, les deux espèces végétales : la pervenche infectée expérimentalement par le phytoplasme du stolbur et la vigne infectée en plein champ par le phytoplasme du BN, réagissent avec l'AcMc anti-stolbur. En présence de 
Chaps ou de Triton $X 100$, les valeurs sont très inférieures à celles obtenues en l'absence de détergent pour la pervenche infectée et même pour la vigne infectée.

\section{Comparaison avec la FD}

La comparaison de la réactivité des anticorps anti-FD, selon l'hôte végétal infecté (fève, pervenche ou vigne) extrait dans le milieu, est donnée dans la figure $4 c$ et $d$ (Elisa spécifique du phytoplasme de la FD). En l'absence de détergent, seuls les extraits faits à partir de fève et pervenche infectées réagissent fortement en Elisa. En revanche, en présence de Chaps ou de Triton $\times 100$, les trois espèces végétales : fève et pervenche (infectées expérimentalement par le phytoplasme de la FD) ainsi que la vigne (infectée en plein champ), réagissent avec les anticorps anti-FD. II est intéressant de noter que la présence d'un de ces deux détergents dans le tampon d'extraction amplifie les résultats en Elisa sur les extraits de vigne infectée alors qu'elle les diminue sur les extraits de fève et de pervenche. Ces observations confirment des observations antérieures sur vigne et fève (Caudwell et Kuszala, 1992). En présence de Tween 40 les antigènes extraits des trois espèces végétales infectées réagissent. Cependant avec la vigne infectée les valeurs sont plus faibles en présence de Tween 40 qu'en présence de Triton ou de Chaps.

Bien que la comparaison du milieu publié pour l'extraction des antigènes du phytoplasme de la FD (Caudwell et Kuszala, 1992) au milieu proposé dans le présent travail montre que ce dernier milieu est moins favorable pour la FD (données non représentées), les résultats de la figure 4 indiquent qu'un même tampon d'extraction (contenant du Tween 40) peut être utilisé pour la détection de ces deux maladies.

La figure 5, qui compare les tests Elisa spécifiques FD et Elisa spécifiques stolbur sur les mêmes extraits de plante, confirme qu'un même tampon peut détecter les deux phytoplasmes dans la vigne. Les valeurs en absorbance des extraits de vigne infectée indiquent que les réactions sont spécifiques de chaque maladie. Les valeurs obtenues en Elisa-FD d'une part et en Elisa-stolbur d'autre part, avec l'échantillon simulant une double infection, permettent une détection différentielle des deux maladies dans le même échantillon extrait en présence de Tween 40.

\section{Origine géographique des jaunisses de la vigne de type $B N$}

Le bilan des tests Elisa spécifiques du phytoplasme du stolbur en fonction des différentes origines géographiques est résumé dans le tableau $I$. Le test a été positif avec des échantillons de France (Alsace et Bourgogne) ; d'Italie du Nord, de Sicile, ainsi que des échantillons de Suisse.

\section{DISCUSSION ET CONCLUSION}

Les essais de cette étude ont prouvé l'existence d'une parenté sérologique entre le phytoplasme du stolbur souche $C$ Versailles et l'agent associé à une jaunisse de type $B N$, et l'absence de parenté sérologique avec l'agent de la FD. De ce fait, l'AcMc anti-stolbur devient un outil pour la détection du $\mathrm{BN}$ et pour son suivi épidémiologique.

Le choix d'un détergent (Shechter, 1990), pour la solubilisation d'une protéine antigénique associée au BN, a été basé sur l'observation expérimentale de la conservation de l'activité de l'épitope reconnu par l'AcMc. Pour une protéine membranaire, son extraction implique tout d'abord

Tableau I. Bilan (exprimé en nombre de ceps positifs sur le nombre total testé) des essais sérologiques Elisa spécifiques du phytoplasme du stolbur sur des échantillons de vigne présentant des symptômes de jaunisse attribuée au BN.

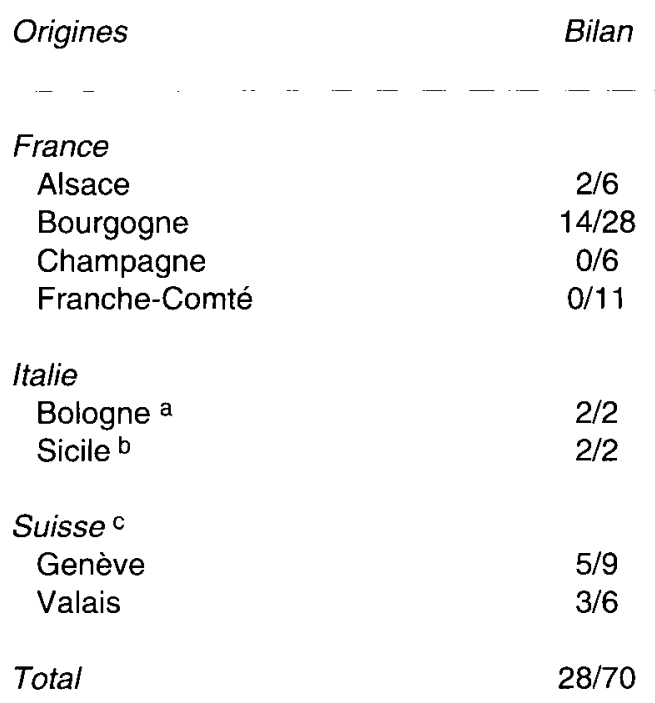

Fournis par a R Credi ; b G Granata ; c O Cazelles. 
une rupture des interactions entre cette protéine et les autres constituants membranaires (lipides, autres protéines), puis son maintien en solution. En présence d'un détergent, les protéines hydrophobes des membranes du phytoplasme sont solubilisées et présentes dans les surnageants. Ceci n'est pas le cas en l'absence de détergent où les protéines membranaires ne sont pas solubilisées. De plus en l'absence de détergent, les protéines hydrophobes extraites en milieu aqueux ont tendance à s'agréger, à se dénaturer et à précipiter de manière irréversible.

La détection en Elisa du BN, du stolbur et de la FD avec un même tampon sans détergent, a mis en évidence une importante différence de réactivité selon l'hôte végétal. Cette différence pourrait s'expliquer par l'action de protéases qui, en l'absence de détergent, hydrolysent l'antigène au cours de la préparation des échantillons. Cette libération de protéases peut être différente d'une espèce végétale à l'autre.

La différence de réactivité en présence de différents détergents peut s'expliquer par leur différence de capacité à solubiliser la protéine membranaire à partir d'un hôte végétal donné. De plus, pour une protéine donnée, les détergents utilisés peuvent avoir une action dénaturante plus ou moins grande. Ainsi donc les conditions expérimentales d'extraction et de solubilisation qui permettent la réaction sérologique varient suivant la nature de la protéine antigénique et de l'extrait végétal dans lequel elle se trouve.

Comme pour le Tween, la présence de PVP 10 a pour effet d'améliorer l'extraction de l'antigène du BN. Elle pourrait participer à sa protection. Elle a l'inconvénient de rendre la solution visqueuse à forte concentration.

Dans l'étude des autres constituants du tampon (données non représentées), nous n'avons pas apporté d'amélioration significative des résultats en remplaçant le tampon Tris par du $\mathrm{K}_{2} \mathrm{HPO}_{4}$ à $250 \mathrm{mM}$, ni en supprimant le $\mathrm{NaCl}$. En revanche nous avons obtenu des valeurs légèrement plus fortes en l'absence d'acide ascorbique. Le pH optimum a été défini à 8,2.

Il est important de souligner l'existence de sources de fluctuations. La vigne est une plante dans laquelle les phytoplasmes se multiplient peu et sont irrégulièrement distribués, aussi est-il primordial de bien sélectionner les prélèvements à tester sur la plante.

L'analyse en Elisa de 70 pieds de vigne présentant des symptômes de jaunisse mais néga- tifs en Elisa spécifique FD a donné 28 réponses positives avec l'anticorps anti-stolbur. Parmi ces 28 cas, la reproductibilité a été vérifiée a plusieurs reprises pour 11 pieds de vigne. En particulier pour trois d'entre eux l'antigène était généralisé sur la totalité du rameau récolté. Les témoins sains de vigne procurés par l'Entav ont toujours réagi négativement. Parmi les autres cas de réponse négative, 17 pieds de vigne négatifs (pour la FD et pour le BN) sont issus des mêmes vignobles (Champagne et FrancheComté). Il est difficile de dire si la concentration en antigène était inférieure à la limite de sensibilité ou s'il s'agit d'une autre cause. Toutefois, ces résultats négatifs, provenant de quelques vignobles, ne permettent pas d'exclure la présence du BN dans ces mêmes régions. Les autres cas négatifs correspondent, dans la plupart des cas, à des pieds de vigne provenant de mêmes vignobles que des pieds positifs. Dans ce cas, l'hypothèse d'une concentration insuffisante en antigène est la plus probable. Pour accroitre la sensibilité du diagnostic tout en conservant une bonne spécificité, l'utilisation de la PCR avec des amorces spécifiques est alors nécessaire.

Contrairement aux tests Elisa, l'utilisation des sondes (Kirkpatrick et al, 1987 ; Garnier et al, 1991) n'était possible que dans des laboratoires spécialisés. Désormais la PCR, réalisable dans la plupart des laboratoires a permis d'augmenter considérablement la sensibilité du diagnostic. Cependant les premières amorces ribosomiques universelles de phytoplasmes ne présentaient pas de spécificité. À l'heure actuelle, il en existe de spécifiques obtenues à partir de régions non conservées de l'ADN ribosomique. De plus, plusieurs laboratoires signalent l'obtention de sondes génomiques (Deng et Hiruki, 1990 ; Daire et al, 1992 ; Jaraush et al, 1994 ; Saeed et al, 1994).

II est intéressant d'étudier la complémentarité des méthodes immunologique et de biologie moléculaire. Par exemple dans le cas de la phyllodie de la féverole et le chanvre du Bengale (Saeed et al, 1994 ; Saeed et Cousin, 1995), les résultats obtenus tendent à montrer l'avantage de la méthode immunologique en ce qui concerne la spécificité (absence de parenté sérologique). Dans le cas de la FD et de vigne et la jaunisse de l'orme (classées dans le même groupe ribosomique) l'hybridation ADN-ADN ne permet pas de les distinguer (Daire et al, 1992). La comparaison des deux phytoplasmoses en Elisa, avec des antigènes immunisants $F D$, a mis en 
évidence successivement : une non-réactivité suggérant une absence de parenté (mentionnée par Daire et al, 1993b), une réactivité mettant en évidence une parenté sérologique (Kuszala, données non publiées), des différences de réactivité selon les souches (Seddas et al, 1996). De plus, chez l'agent pathogène de la $\mathrm{FD}$, des antigènes détectés à un stade de développement donné peuvent différer de ceux détectés à un autre stade (Kuszala et al, 1990). Les résultats obtenus tendent à montrer l'avantage de la méthode immunologique pour l'étude de la spécificité et le stade de l'infection. En revanche sa sensibilité est moindre. Dans certains cas, seules les méthodes de biologie moléculaire restent utilisables en raison de la faible concentration de la plante en phytoplasmes.

Récemment, deux paires d'amorces spécifiques (à partir de l'ADN non ribosomique) du stolbur $Y$ (isolat croate) ont été obtenues et utilisées pour la détection du BN sur la vigne (Daire et al, 1996). Dans ces conditions il serait intéressant de comparer les deux approches, sérologique et moléculaire, en fonction des finalités recherchées, du matériel à l'étude (plantes herbacées riches en phytoplasmes ou plantes ligneuses souvent peu concentrées) et des possibilités des laboratoires concernés.

Ce travail a montré pour la première fois que l'agent associé au BN est antigéniquement semblable au stolbur $\mathrm{C}$ et est présent dans différentes régions. Selon Fos et al, 1992, l'anticorps anti-stolbur reconnaît des isolats de stolbur d'origines diverses. D'autre part, le stolbur est un phytoplasme très répandu, présent dans de nombreuses espèces végétales. Selon Daire (communication personnelle), peu de polymorphisme a été trouvé dans les différents fragments d'ADN génomiques de ce phytoplasme, aussi bien dans les vignes atteintes de $B N$ de différents pays, que dans des isolats sur pervenche de phytoplasmes classés dans le sous-groupe stolbur du groupe aster yellows (Seemüller et al, 1994).

En conclusion, l'intérêt immédiat de ce travail est un diagnostic différentiel du BN avec la FD, que l'on ne peut pas faire sur la base des symptômes.

\section{REMERCIEMENTS}

Nous remercions Sanofi Diagnostic Pasteur, département Phytodiagnostic (A Lefèbvre, F Poul et JM
Clauzelle) pour la mise à disposition du réactif antistolbur ; A Pugin (université de Bourgogne) pour ses conseils sur le rôle des détergents; E Boudon-Padieu (Inra-Dijon) pour nos discussions fructueuses et la mise à la disposition des sérums polyclonaux anti-FD ; $\mathrm{R}$ Meignoz et $\mathrm{A}$ Seddas (Inra-Dijon) pour les lignées de monoclonaux anti-FD.

\section{RÉFÉRENCES}

Boubals D (1993) Une grave épidémie de jaunisse de la vigne dans le Golan (Israël). Prog Agric Vitic 17, 361-364

Boudon-Padieu E, Larrue J, Caudwell A (1989) ELISA and Dot-Blot detection of Flavescence dorée MLO in individual leafhopper vectors during latency and inoculative state. Curr Microbiol 19, 357-364

Caudwell A (1961) Étude sur la maladie du bois noir de la vigne: ses rapports avec la flavescence dorée. Ann Epiphyt 12, 241-262

Caudwell A, Ottenwaelter M (1957) Deux années d'étude sur la flavescence dorée, nouvelle maladie grave de la vigne. Ann Amelior Plant 7, 359-395

Caudwell A, Kuszala C (1992) Mise au point d'un test Elisa sur les tissus de vignes atteintes de flavescence dorée. Res Microbiol 143, 791-806

Caudwell A, Giannotti J, Kuszala C, Larrue J (1971a) Étude du rôle de particules de type «mycoplasme» dans l'étiologie de la flavescence dorée de la vigne. Examen cytologique des plantes malades et des cicadelles infectieuses. Ann Phytopathol 3, 107-123

Caudwell A, Larrue J, Kuszala C, Bachelier JC (1971b) Pluralité des jaunisses de la vigne. Ann Phytopathol 3, 95-105

Caudwell A, Meignoz R, Kuszala C, Schneider C, Larrue J, Fleury A, Boudon E (1982) Serological purification and visualization in the electromicroscope of the grapevine Flavescence dorée pathogen, in infectious vectors extracts and in diseased plants extracts. Yale J Biol Med 65, 936-937

Cazelles O, Kuszala C (1993) Prospection des jaunisses de la vigne en Suisse romande et au Tessin et comparaison à la FD par le test Elisa. Rev Suisse Vitic Arboric Hortic 25, 4, 257-259

Cazelles O, Desbaillet C, Schmid A (1992) Jaunisses de la vigne en Suisse romande et au Tessin. Rev Suisse Vitic Arboric Hortic 24, 133-134

Clark MF, Davies DL, Buss SL, Morton A (1988) Serological discrimination among mycoplasma-like organisms using polyclonal and monoclonal antibodies. Acta Hortic 235

Cousin MT (1995) Phytoplasmes et phytoplasmoses. agronomie 15, 245-264

Cousin MT, Grison C (1966) Quelques observations et essais concernant le stolbur de la tomate. Ann Epiphyt 17, (HS), 99-111 
Cousin MT, Grison C, Decharme M (1968) Étude comparée de plusieurs types de flétrissements de solanacées. Polyphagie du virus du stolbur. Ann Epiphyt 19, (HS), 121-140

Cousin MT, Dafalla G, Demazeau E, Theveu E, Grosclaude J (1989) In situ detection of MLOs for solanaceae stolbur and faba bean phyllody by indirect immunofluorescence. J Phytopathol 124, 7179

Credi R, Santucci A (1992) Dodder transmission of mycoplasmalike organism (MLOs) from grapevines affected by a Flavescence dorée-type disease to periwinkle. Phytopathol Mediterr 31, 154-162

Daire X (1994) Détection et différenciation de mycoplasma-like organisms (MLO) associés aux maladies de la vigne de type jaunisse. Thèse doctorat, université de Bourgogne

Daire X, Boudon-Padieu E, Berville A, Scheider B, Caudwell A (1992) Cloned DNA probes for detection of grapevine Flavescence dorée mycoplasmalike organism (MLO). Ann Appl Biol 121, 95-103

Daire X, Kuszala C, Larrue J, Caudwell A, Magnien C, Boulud J (1993a) Les jaunisses de la vigne, flavescence dorée, bois noir, etc, en Bourgogne et dans les régions voisines. Le Progrès Agric Vitic 8

Daire X, Clair D, Larrue J, Boudon-Padieu E, Caudwell A (1993b) Diversity among mycoplasma-like organisms inducing grapevine yellows in France. Vitis $32,159-163$

Daire X, Clair D, Larrue J, Boudon-Padieu E, Alma A, Arzone A, Carraro L, Osler R, Refatti E, Granata G, Credi R, Tanne E, Pearson R, Caudwell A (1993c) Occurrence of diverse MLOs in tissues of grapevine affected by grapevine yellows in different countries. Vitis $32,247-248$

Daire X, Clair D, Larrue J, Boudon-Padieu E (1996) Enquête sur les jaunisses à phytoplasmes de la vigne dans différents pays. $2^{\text {es }}$ Rencontres de phytobactériologie, Aussois, France, 38

Deng S, Hiruki C (1990) Enhanced detection of a plant pathogenic mycroplasma-like organism by polymerase chain reaction. Proc Jpn Acad 66, B, 140-144

Fos A, Danet JL, Zreik L, Garnier M, Bove JM (1992) Use of a monoclonal antibody to detect the stolbur mycoplasmalike organism in plants and insects and to identify a vector in France. Plant Dis 76, 11

Garnier M, Martin-Gros G, Iskra ML, Zreik L, Gandar J, Fos A, Bové JM (1990) Monoclonal antibodies against the MLOs associated with tomato stolbur and clover phyllody. Zentrabl Bakteriol 20 (Suppl) 263-269

Garnier M, Zreik L, Bové JM (1991) Witches' Broom, a lethal mycoplasmal disease of lime trees in the Sultanate of Oman and the United Arab Emirates. Plant Dis 75, 6, 546-551

Gärtel W (1965) Untersuchungen über das Auftreten und das Verhalten des flavescence dorée in den Weinbaugebieten an Mosel und Rhein. Weinberg Keller 12, 347-376
Gourret JP (1971) Évolution des plastes dans les végétaux infectés par des mycoplasmes : problèmes de la jaunisse et des pétales verts. Physiol Vég 9, 583-594

Granata G, Grimaldi V (1991) Electron microscopic detection of mycoplasma-like organisms in epidemic yellow affected grapevines. Petria 1, 171-175

Gugerli P (1984) Une méthode simple pour le broyage de tissu végétal. Rev Suisse Vitic Arboric Hortic 16 , 87-88

Jarausch W, Saillard C, Dosba F, Bové JM (1994) Differentiation of mycroplasmalike organism (MLOs) in European fruit trees by PCR using specific primers derived from the sequence of a chromosomal fragment of the apple proliferation MLO. App/ Environ Microbiol 2916-2923

Kirkpatrick B, Stenger DC, Morris TJ, Purcell AH (1987) Cloning and detection of DNA from a nonculturable plant pathogenic mycoplasma-like organism. Science 238, 197-200

Kirkpatrick B, Smart C, Gardner S, Gao JL, Ahrens U, Maurer R, Schneider B, Lorenz KH, Seemuller E, Harrison N, Namba S, Daire X (1994) Phylogenic relationships of plant pathogenic MLOs established by $16 / 23 S$ rDNA spacer sequences. 10th Int Congress IOM Letters, France 3, 228-229

Kuszala C, Meignoz R, Caudwell A (1990) Evolution of Flavescence dorée MLO antigens according to the age of infection. 10th Meeting ICVG Volos, Greece, Proc, 484-487

Kuszala C, Cazelles O, Boulud J, Credi R, Granata G, Kriel G, Magarey P, Magnien C, Pearson RC, Refatti E, Tanne E, Caudwell A (1993) Contribution à l'étude des jaunisses de la vigne dans le monde: prospection par test Elisa spécifique du MLO de la flavescence dorée. agronomie 13, 929-933

Magarey PA, Wachtel MF (1986) Australian grapevine yellows. Int J Trop Plant Dis 4, 1-14

Maixner M (1994) Transmission of German grapevine yellow's (Vergilbungskrankheit) by the planthopper Hyalesthes obsoletus (Auchenorrhyncha: Cixiidae). Vitis 33, 103-104

Maixner M, Ahrens U, Seemüller E (1994) Detection of mycoplasmalike organisms associated with a yellows disease of grapevine in Germany. J Phytopathol 142, 1-10

Pearson RC, Pool RM, Gonsalves D, Coffinet MC (1985) Occurrence of Flavescence dorée-like symptoms on 'White Riesling' grapevines in New York, USA. Phytopathol Mediterr 24, 82-87

Saeed E, Cousin MT (1995) The genetic relationship between MLOs causing diseases in the Sudan and different continents revealed by polymerase chain reaction (PCR) amplification of the 16S RNA gene followed by RFLP analysis. J Phytopathol 143, 1720

Saeed E, Seemüller E, Schneider B, Saillard C, Blanchard B, Bertheau Y, Cousin MT (1994) Molecular cloning, detection of chromosomal DNA of the mycoplasma-like organism (MLO) associated 
with faba bean (Vicia faba L) phyllody by southern blot hybridization and the polymerase chain reaction (PCR). J Phytopathol 142, 97-106

Schwartz Y, Boudon-Padieu E, Grange J, Meignoz R, Caudwell A (1989) Obtention d'anticorps monoclonaux spécifiques de l'agent pathogène de type mycoplasme de la flavescence dorée de la vigne. Res Microbiol 140, 311-324

Schwester D, Carle P, Moutous G (1963) Transmission de la flavescence dorée de la vigne par Scaphoideus titanus Ball. Ann Epiphyt 14, 175-198

Sears BB, Schewe G (1994) Optimization of growth conditions for Acholeplasma strains J233 as a strategy for culturing phytoplasmas in vitro. 10th Int Congress IOM Letters, France 3, 291-292

Seddas A, Meignoz R, Daire X, Boudon-Padieu E (1996) Generation and characterization of monoclonal antibodies to Flavescence dorée phytoplasma: serological relationships and differences in electroblot immunoassay profiles of Flavescence dorée and elm yellows phytoplasmas. Eur J Plant Pathol (sous presse)

Seemüller E, Schneider B, Mäurer R, Ahrens U, Daire $X$, Kison H, Lorenz KH, Firrao G, Avinent L, Sears BB, Stackebrant E (1994) Phylogenetic classification of phytopathogenic mollicutes by sequence analysis of $16 \mathrm{~S}$ ribosomal DNA. Int J Syst Bacteriol $44,3,440-446$

Shechter E (1990) Biochimie et biophysique des membranes. Aspects structuraux et fonctionnels. Masson, Paris

Valenta V, Musil M, Misiga S (1961) Investigations on European Yellows-type virus I. The stolbur Virus. Phytopathol Z 42, 1-38

Zreik L, Carle P, Bové JM, Garnier M (1995) Characterization of the mycoplasmalike organism associated with witches'-broom disease of lime and proposition of a candidatus taxon for the organism, 'candidatus phytoplasma aurantifolia' Int $J$ Syst Bacteriol 45, 3, 449-453 